\title{
DECISIONS
}

\section{A 32-year-old woman with headache}

\author{
William Jeptha Davenport MD, Tamara M. Pringsheim MD MSc
}

\begin{abstract}
A 32-year-old woman presents to her primary care physician about a headache she experienced a week ago. She states that the pain began behind one eye and was accompanied by nausea and photophobia. Over a few hours, the pain spread to involve the temple and occiput unilaterally. It also intensified, prompting the patient to leave work early. Once home, she took two 500-mg tablets of acetaminophen but vomited shortly afterward. She lay down despite the early hour and eventually fell asleep.
\end{abstract}

\section{What questions should this patient be asked?}

A unilateral, pulsatile headache of moderate to severe intensity that worsens with exertion and is accompanied by nausea or vomiting, or by photophobia and phonophobia, suggests migraine. The clinician should ask about additional historical features of the headache that may be consistent with migraine, including the quality (pulsatile) and duration ( $>4 \mathrm{~h}$ to $72 \mathrm{~h}$ if untreated) and whether the patient has had similar episodes previously. Diagnostic criteria for migraine stipulate five attacks, but when fewer episodes have occurred, a diagnosis of probable migraine may be entertained. ${ }^{1}$

The clinician should ask about worrisome features of headache, or red flags, that could indicate a cause other than migraine (Box 1). ${ }^{2}$

When present, a fully reversible, unilateral aura, spreading and lasting from 5 to 60 minutes in close temporal association with head pain, suggests migraine with aura. ${ }^{1}$ In isolation, certain aura symptoms (e.g., aphasia and hemiparesis) may resemble stroke, distress patients and confound clinicians. Although the affected tissue is the same in each case, the cause and course are not.

\section{What should be included on physician examination?}

Findings on physical examination should be normal or at the patient's baseline between migraine attacks. Expert opinion, guidelines and nonrandomized comparative studies suggest that a screening neurologic examination be performed. ${ }^{2,3}$ It should include a general assessment of the patient's mental status, cranial nerve examination (in particular, confrontation visual field test, funduscopy, pupillary symmetry and reactivity, eye movements and facial symmetry), assessment of strength, coordination and reflexes in the limbs, and gait. ${ }^{3}$ The neurologic examination serves to rule out secondary causes of headache, such as mass lesions that can produce focal neurologic deficits or signs of raised intracranial pressure. A more detailed neurologic examination is warranted when deficits are identified.

Examination of the range of motion of the neck and palpation for tenderness is recommended to rule out meningismus and cervical arthritic changes that can produce cervicogenic headache. ${ }^{3}$ Examination of the jaw for range of motion and tenderness is also recommended

\section{Box 1: Red-flag symptoms and signs in headache (and possible causes of secondary headache) ${ }^{2}$}

Emergent evaluation in an emergency department, including cranial imaging and lumbar puncture if deemed appropriate by the physician, is recommended in the presence of the following features on history-taking or physical examination:

- Thunderclap headache, with severe pain of rapid onset reaching maximum intensity within seconds to five minutes (subarachnoid or intracranial hemorrhage, arterial dissection, pituitary apoplexy)

- Pain accompanied by an immediate or persisting neurologic deficit (stroke, arterial dissection)

- Fever or meningismus (encephalitis, meningitis)

- Focal infection of the ears, nose or throat (abscess with intracranial extension)

- History of cancer, immunosuppression or a clotting disorder (metastasis, opportunistic infection, cerebral venous sinus thrombosis)

- Pain precipitated by trauma, Valsalva manoeuvre or change in posture (intracranial hemorrhage or mass)

Urgent laboratory evaluation of the erythrocyte sedimentation rate and C-reactive protein level, and referral for temporal artery biopsy if results are abnormal, is recommended in the presence of:

- Jaw pain, visual disturbance or both (giant cell arteritis)

Outpatient cranial imaging, with computed tomography at a minimum, is recommended for:

- Patients presenting nonurgently with a first or new headache past age 50 years 
when the patient's history suggests pathology in that region, such as temporal mandibular joint dysfunction or temporal arteritis. ${ }^{3}$

\section{Are any investigations required?}

If the patient's history meets the criteria for migraine, no red-flag signs or symptoms are identified (Box 1), and the results of the neurologic examination are normal, no further investigations are required. ${ }^{3}$ In a prospective neuroimaging study (computed tomography or magnetic resonance imaging) involving people with nonacute headache referred for neurologic consultation, only $4(0.4 \%)$ of 920 patients who met the diagnostic criteria for migraine had clinically significant abnormalities on neuroimaging. ${ }^{4}$

\section{What treatments should be considered?}

Once aware of the diagnosis, most patients with migraine choose and respond well to acute (abortive) pharmacologic therapy. When medications such as acetaminophen, ibuprofen or acetylsalicylic acid fail to provide adequate pain relief, systematic reviews of randomized controlled trials have shown that triptans are effective in aborting the pain of migraine attacks, especially if used early (within 30 min after onset of pain). ${ }^{5}$

Seven triptans are available by prescription in Canada. The number needed to treat for a painfree response at two hours is 4.7 for sumatriptan 100 mg. ${ }^{6}$ A Canadian clinical practice guideline based on expert consensus recommends that each triptan be assayed in turn at separate attacks until one is identified to be frequently effective for a particular patient. ${ }^{7}$ For patients who are unable to take oral medications because of severe nausea, a nasal spray formulation is available for sumatriptan and zolmitriptan, and a subcutaneous self-injection formulation is available for sumatriptan.

Many patients with migraine are aware of factors that may increase the risk of a migraine attack. These triggers vary from patient to patient and include poor sleep, hunger, psychophysical stressors, and certain foods and substances. When the frequency of migraine attacks interferes with work and other activities, lifestyle modification and drug prophylaxis (e.g., $\beta$-blocker or tricyclic antidepressant) are options to explore. ${ }^{8}$

\section{When should the patient be referred?}

Referral to a headache clinic, neurologist or physician with expertise in headache management may benefit patients who have frequent migraines (more than twice per week) despite lifestyle modification, trigger avoidance and drug prophylaxis; those who do not respond to commonly used treatments such as triptans; those who have other less common primary headaches that are not well controlled (e.g., trigeminal neuralgia, cluster headache or hemiplegic migraine); and patients who have atypical headaches (headaches that do not appear to meet diagnostic criteria for primary headache disorder). ${ }^{3}$

\section{Case revisited}

When the patient awoke the day after the initial headache, her head pain was mild. She returned to work, feeling fatigued but capable of her usual activities. At the visit with her physician, she reported having similar episodes previously, beginning around age 27. No red-flag symptoms or signs were identified. Findings on a screening neurologic examination and neck examination were normal. The patient's physician determined that her condition met the criteria for episodic migraine without aura.

The patient was given information about migraine diagnosis, possible lifestyle factors related to migraine occurrence and the option of abortive treatment strategies. For her next migraine, she was advised to take $500 \mathrm{mg}$ of naproxen, and $10 \mathrm{mg}$ of metoclopramide if nausea was a prominent feature. If this strategy failed to provide adequate and timely relief, she would be given a prescription for an oral triptan medication to try for the next attack.

\section{References}

1. Headache Classification Committee, International Headache Society. The international classification of headache disorders, 3rd edition (beta version). Cephalalgia 2013;33:629-808.

2. Diagnosis and management of headache in adults: a national clinical guideline. Edinburgh: Scottish Intercollegiate Guidelines Network; 2008. Available: www.sign.ac.uk/pdf/sign107. pdf (accessed 2016 Jan. 23).

3. Guideline for primary care management of headache in adults. Edmonton: Toward Optimized Practice and Institute of Health Economics; 2012. Available: http://topalbertadoctors.org/ cpgs/10065 (accessed 2016 Jan. 23).

4. Sempere AP, Porta-Etessam J, Medrano V, et al. Neuroimaging in the evaluation of patients with non-acute headache. Cephalalgia $2005 ; 25: 30-5$.

5. Valade D. Early treatment of acute migraine: new evidence of benefits. Cephalalgia 2009;29(Suppl 3):15-21.

6. Derry C, Derry S, Moore R. Sumatriptan (oral route of administration) for acute migraine attacks in adults. Cochrane Database Syst Rev 2012;(2):008615.

7. Worthington I, Pringsheim T, Gawel MJ, et al. Canadian Headache Society guideline: acute drug therapy for migraine headache. Can J Neurol Sci 2013;40(Suppl 3):S1-80.

8. Pringsheim T, Davenport WJ, Becker WJ. Prophylaxis of migraine headache. CMAJ 2010;182:E269-76.

Affiliation: Department of Clinical Neurosciences, University of Calgary, Calgary, Alta.

Contributors: William Jeptha Davenport drafted the manuscript, which both authors revised. Both authors gave final approval of the version to be published and agreed to act as guarantors of the work. 